\title{
Faster Time-Resolved Electrostatic Force Microscopy
}

\author{
Durmus U. Karatay ${ }^{1,2}$, Jeffrey S. Harrison ${ }^{2}$, Micah Glaz ${ }^{2}$, Phillip A. Cox ${ }^{2}$ and David S. Ginger ${ }^{1,2}$ \\ 1. Department of Physics, University of Washington, Seattle, WA 98105 \\ 2. Department of Chemistry, University of Washington, Seattle, WA 98105
}

Electrostatic force microscopy (EFM) is a widely used technique to study systems with nanoscale spatial resolution in various fields of science. Different EFM methods have been utilized to study phenomena such as mapping local electrical properties of graphene and measuring dielectric constants [1,2]. However, classic EFM methods usually do not capture time-domain information or, when they do, integrate over long times - on the order of minutes to hours.

Our group has demonstrated that it is possible to perform time-resolved EFM (trEFM) to capture dynamic information, first on timescales as fast as $100 \mu \mathrm{s}$ [3], and more recently as fast as $100 \mathrm{~ns}$ [4], and we have shown that these methods can provide important information about electronic processes such as photocurrent generation in devices with and without nanostructures.

However, while offering impressive time-resolution, these methods are often slow in image acquisition. Here, we report the recent advances and findings regarding feedback-free trEFM.

The motion of most AFM cantilevers can be modeled by damped driven harmonic oscillator equation:

$$
\frac{d^{2} z}{d t^{2}}+2 \beta \frac{d z}{d t}+\omega_{0}(t)^{2} z=\frac{F_{0}}{m} \cos (\omega t)+\frac{F_{e}(t)}{m}
$$

where $z$ is displacement of the tip, $F_{0}$ is the driving force, $m$ is the effective cantilever mass, $\omega_{0}$ is the resonance frequency of the cantilever and $F_{e}$ is an external force, which can be modeled as an exponential decay:

$$
F_{e}(t \geq 0) \cong F_{e}(\infty)\left[1-\mathrm{e}^{-t / \tau}\right]
$$

Using signal processing methods we can extract the change in the instantaneous frequency of the cantilever which is related to the characteristic time of an exponentially decaying excitation. However, the phase of the cantilever at trigger time affects the cantilever response immensely. In order to quantitatively study the effects of phase on the data extraction, we have done experiments with a gold standard and voltage pulses ranging from $10 \mathrm{~ns}$ to $1000 \mathrm{~ns}$. The resulting instantaneous frequencies due to triggering at different phases at $\tau=100 \mathrm{~ns}$ can be seen in Figure 1.a.

It is clear that some phases, namely $0^{\circ}$ and $270^{\circ}$, cause non-ideal responses. Triggering at $180^{\circ}$ gives the best response curves. In Figure 1.b, we show the time resolution of our equipment for a $300 \mathrm{kHz}$ cantilever. We are able to differentiate signals with $\tau=100 \mathrm{~ns}$, which puts our time-resolution at $100 \mathrm{~ns}$.

We also have developed a real-time imaging system for feedback-free trEFM, where we can image the topography and the charging rate of the material at the same time. Currently, for a $128 \times 128$ image, it takes $\sim 1$ hour.

In Figure 2, we show an image of an organic photovoltaic film composed of a polymer MDMO-PPV 
mixed with fullerene-derivative PCBM, taken using feedback-free trEFM. It can be clearly seen that the charging rate is dependent on local film morphology.
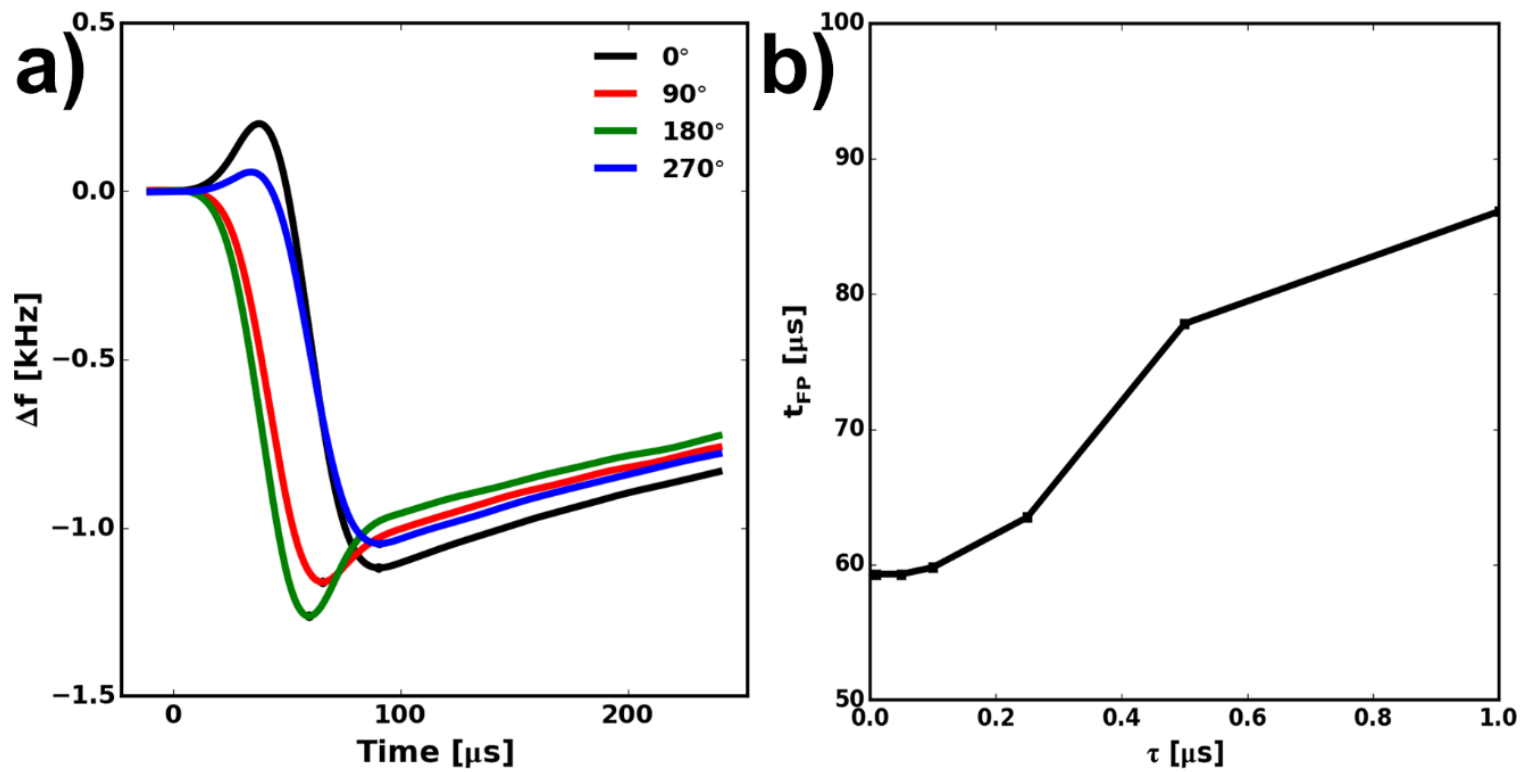

Figure 1. (a) Deviation of cantilever's instantaneous frequency from the drive frequency for an exponential voltage pulse with $\tau=100 \mathrm{~ns}$, (b) Resolution of the FF-trEFM for different values of $\tau$ at trigger phase of $180^{\circ}$. Both results are for a $300 \mathrm{kHz}$ cantilever.
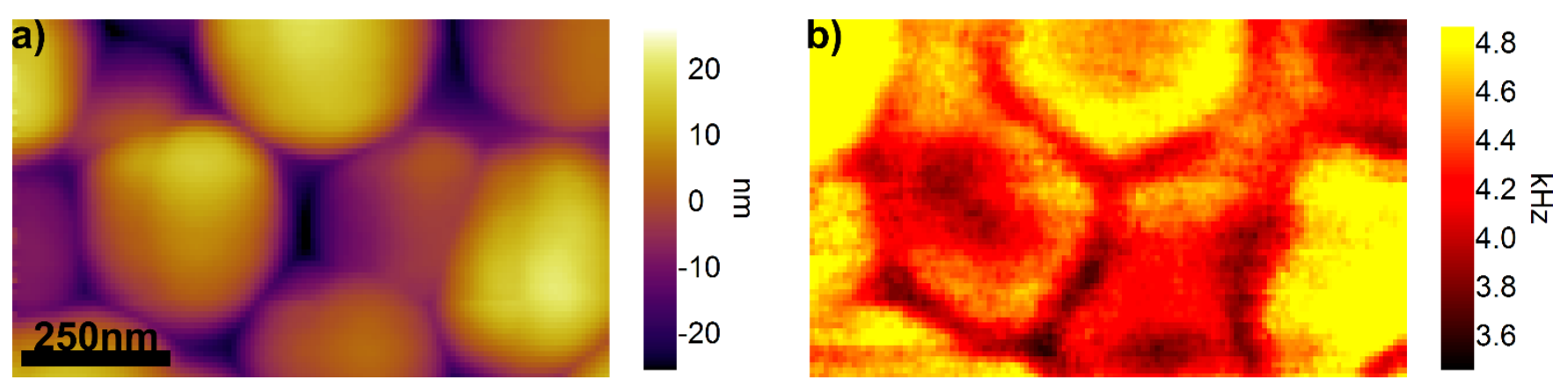

Figure 2. (a) Topography and (b) charging rate measurements for an MDMO-PPV:PCBM film.

References:

[1] T. Burnett et al., Nano Lett. 11 (2011) 2324.

[2] E. Castellano-Hernández et al., J. Phys. Condens. Matter 24 (2012) 155303.

[3] D.C. Coffey, D.S. Ginger, Nat. Mater. 5 (2006) 735.

[4] R. Giridharagopal et al., Nano Lett. 12 (2012) 893.

[5]The authors acknowledge funding from NSF MRI DMR-1337173. 